\title{
3D temperature map reconstruction of a ceramic shell mold in investment casting process
}

\author{
by C.S. Greco, G. Paolillo, C. Caramiello, M. Di Foggia and G. Cardone
}

\author{
University of Naples Federico II, 80125, Piazzale Tecchio 80, Naples, Italy, carlosalvatore.greco@unina.it
}

\begin{abstract}
This study analyzes the thermal behaviour of a ceramic shell mold during the a stage of the investment casting process. The investment casting process is a multi-step procedure which allows producing very reliable metal components such as turbine blades. Many parameters influence the final product of an investment casting process such as the initial mold temperature. This initial thermal status strongly affects the radiative heat transfer during the casting and, consequently, the product quality. This work describes and discusses the 3D temperature distribution over the ceramic shell mold of a turbine blade.
\end{abstract}

\section{Introduction}

The investment casting is a multi-step process used to produce components of excellent surface finish, dimensional accuracy and complex shape. Fundamentally, this process consists of making wax patterns by injecting wax into metal die, building of ceramic shell mold around the wax patterns, dewaxing of ceramic shell mold, and casting of alloys into the shell mold itself [1]. During the process many are the parameters which influence the quality of the final component. Indeed, according to Shen et al. [2], the quality of the final part is strictly related to injection process parameters such as cooling time, injection pressure, melt temperature, injection speed, injection time, filling time and mold temperature.

Many industrial components are produced by using the investment casting process such as jewellery/art castings, turbine blades and other industrial components. Turbine blades are mainly used in aero engines and gas turbines and have very 3D complex geometries. Indeed the non-uniform thickness of airfoil, thick root, curvature and twist are critical features of this element. Due to their nature, it is difficult to machine this shape of the blades. Moreover gas turbine blades have narrow dimensional and geometrical tolerances and, for this reason, the investment casting is the best suitable process for their production.

During this process, the ceramic shell has to have a high degree of confidence because the cost incurred when the shell fails during casting can be unacceptable both in terms of plant downtime as well as material loss [3]. For this reason, the ceramic shell mold should fulfill certain requirements in order to cast the required component successfully [4].

The process before the final casting consists in preheating the ceramic shell in a medium-temperature furnace and then bringing this preheated mold to the high-temperature vacuum furnace. During this transportation, the convective and radiative heat transfer cause a drastic change of the mold temperature. It is worth to highlight that, before the casting begins, the initial temperature of the ceramic shell mold is different to what set in the preheating furnace. Moreover the temperature distribution of the mold is non-uniform because of the different convective and radiative heat transfer caused by the highly three dimensional geometry. The mold temperature is one of the process parameter that influences the product, as also reported in [2].

In literature many studies employ thermocouples $[5,6,7,8]$ in order to measure the external surface temperature and then evaluate the heat transfer. The use of thermocouples allows for an intrusive punctual measurements of the surface temperature in opposition to the IR thermography technique $[9,10]$ which is a non-intrusive optical technique. Nevertheless, the infrared data are in the form of 2D images while the ceramic shell mold is a highly three-dimensional body and a reconstruction of the temperature map is necessary. In literature a methodology to rebuild temperature maps on the surface of 3D objects is presented by Cardone et al. [11]. Their procedure is based on an optical calibration of the IR camera by means of a target plate with control points and takes also into account the directional emissivity by estimating the viewing angle.

The aim of this paper is to reconstruct the 3D temperature map of the ceramic shell mold before the beginning of the casting by using the 3D reconstruction procedure discussed by Cardone et al. [11]. The 3D temperature map is then discussed and analysed in order to find the regions of the mold that more preserve the temperature of the preheating furnace.

The paper has been divided as following: Section 2 describes the theory of the 3D reconstuction, Section 3 shows the optical calibration and the experimental apparatus employed in the present investigation, in Section 4 the results are analyzed and Section 5 draws the final conclusions. 


\subsection{1/qirt.2016.160}

\section{3D Reconstruction theory}

In this section the steps involved in the proposed approach to rebuild temperature maps onto a 3D surface grid from 2D IR images are described:

(1) The choice of the camera model and of the calibration algorithm,

(2) The calibration: the design of the target with IR control points (markers), the span of the investigated volume with the target, the determination of the real-world coordinates of these markers, the identification of the positions of the markers in the image plane and finally the determination of parameters of the camera model (transformation between the real-world markers and their corresponding image locations),

(3) The temperature maps reconstruction: the generation of a grid representative of the observed surface, the application of the camera model to the points of the grid to obtain the corresponding measured intensities, the evaluation of the viewing angle and the directional emissivity on the points of the grid and the computation of the surface temperature with the use of the calibration law on the grid points.

\subsection{Camera calibration model}

The camera model used in this work is based on perspective projection with a model for lens distortion [12].

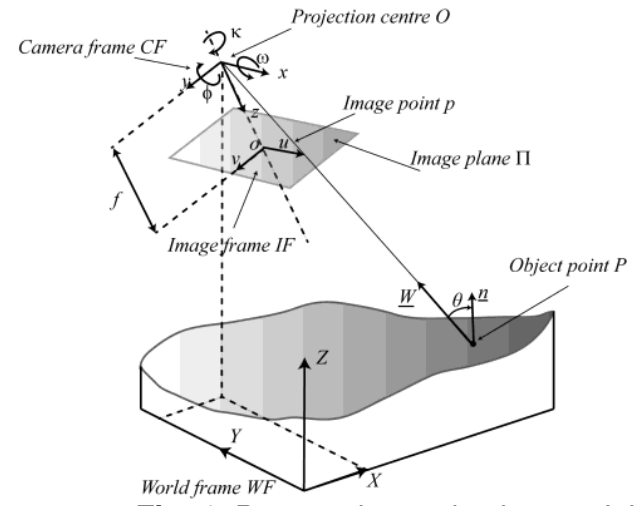

Fig. 1. Perspective projection model.

Consider a model of pure perspective projection, illustrated in figure 1 . The centre of projection is the origin $\mathrm{O}$ of the image in the camera frame CF. The image plane $\Pi$ is parallel to the xy plane and is placed at a distance $f$ (focal length) from projection center $O$ along the $z$ axis; the $z$ axis is also called optical axis and the intersection with the plane $\Pi$ is called the principal point $o$. The axes $u$ and $v$ of the $2 D$ image frame IF are parallel to the axes $x$ and $y$, respectively. The coordinates of $o$ in IF are $\left[u_{0}, v_{0}\right]^{\top}$. Let $P$ be any point in the positive portion of $z$, and $p$ its projection on the image plane. The coordinates of $P$ in the world frame $W F$ are $[X, Y, Z]^{\top}$ while in the camera frame $C F$ are $[x, y, z]^{\top}$. The coordinates of $\mathrm{p}$ in IF are $[\mathrm{u}, \mathrm{v}]^{\top}$ and can be given by the transformation:

$$
\left[\begin{array}{l}
u \\
v \\
1
\end{array}\right] \propto\left[\begin{array}{l}
\sigma u \\
\sigma v \\
\sigma 1
\end{array}\right]=\underline{=} \underline{=}\left[\begin{array}{l}
X \\
Y \\
Z \\
1
\end{array}\right]
$$

where $\sigma$ is a scale factor and $\underline{\underline{P}} \underline{\underline{M}}$ is the perspective transformation matrix with:

$$
P=\left[\begin{array}{cccc}
\xi f & 0 & u_{0} & 0 \\
0 & f & v_{0} & 0 \\
0 & 0 & 1 & 0
\end{array}\right]
$$

where $\xi$ is pixel aspect ratio and $\underline{\underline{M}}$ is a $4 \times 4$ matrix describing the transformation from $W F$ to $C F$. The matrix $\underline{\underline{M}}$ is composed of a pure rotation and a translation as reported in Eq. (3). 


$$
\underline{\underline{M}}=\left[\begin{array}{cc}
\underline{\underline{R}} & \underline{t} \\
0 & 1
\end{array}\right]\left[\begin{array}{cccc}
r_{11} & r_{12} & r_{13} & t_{x} \\
r_{21} & r_{22} & r_{23} & t_{y} \\
r_{31} & r_{32} & r_{33} & t_{z} \\
0 & 0 & 0 & 1
\end{array}\right]
$$

where $\underline{t}=\left[t_{x}, t_{y}, t_{z}\right]^{\top}$ describes the translation between the two frames, and $\underline{R}$ is a $3 \times 3$ orthonormal rotation matrix that can be defined by the three Euler angles $\omega, \varphi$, and $k$. The parameters $t_{x}, t_{y}, t_{z}, \omega, \varphi$ and $k$ are called extrinsic parameters or parameters of external projection and the parameters $s, f, u_{0}$ and $v_{0}$ are the intrinsic parameters of the camera or internal projection parameters of the pinhole camera model. It is usually more convenient to express the coordinates of the image in pixels, so the coordinates obtained by Eq. (1) are multiplied by the factors $D_{u}$ and $D_{v}$ that specify the relationship between pixels and the physical units of the object, for example millimetres.

The perspective projection typically is not sufficient to exactly model real cameras. Ideally, light rays coming from the outside should move linearly in the optical centre of the lens, but in practice, the lens systems introduce nonlinear distortions in optical paths and in the resulting images. The perspective projection camera model gives the ideal image coordinates $[u, v]^{\top}$ of the projected point $\mathrm{p}$. In order to distinguish these ideal coordinates error from the equivalent distorted coordinates, henceforth the correct coordinates are denoted with $a_{c}=\left[u_{c}, v_{c}\right]^{\top}$ and the distorted coordinates are denoted with $a_{d}=\left[u_{d}, v_{d}\right]^{\top}$. The approach most commonly used is to decompose the distortion in its radial and tangential parts. If only two radial distortion coefficients $k_{1}$ and $k_{2}$, and only two tangential distortion coefficients $p_{1}$ and $\mathrm{p}_{2}$ are used, the approximation of the inverse distortion model becomes:

$$
\begin{gathered}
a_{d} \approx a_{c}-\Phi_{D} \\
\Phi_{D}=\frac{\left[\begin{array}{l}
\bar{u}_{c}\left(k_{1} r_{c}^{2}+k_{2} r_{c}^{4}\right)+2 p_{1} \overline{u_{c}} \overline{v_{c}} \\
+p_{2}\left(r_{c}^{2}+2 \overline{u_{c}^{2}}\right) \\
\overline{v_{c}}\left(k_{1} r_{c}^{2}+k_{2} r_{c}^{4}\right)+p_{1}\left(r_{c}^{2}+2 \overline{v_{c}^{2}}\right) \\
+2 p_{2} \overline{u_{c} \bar{v}_{c}}
\end{array}\right]}{4 k_{1} r_{c}^{2}+6 k_{2} r_{c}^{4}+8 p_{1} \overline{v_{c}}+8 p_{2} \bar{u}_{c}+1}
\end{gathered}
$$

\subsection{Calibration target and procedure}

The use of the proposed camera model to establish a correspondence between space coordinates and discrete image coordinates implies the need of a calibration process to estimate the parameters to perform this transformation. This purpose is generally accomplished with the help of a calibration target. This is typically a plane surface on which an array of equidistant points in two orthogonal directions is placed; once an origin has been set on this surface and the distance between the points is known, the physical coordinates of each point of the array is known; once an image has been recorded by the camera undergoing calibration, the coordinates of the points will be known also in the image reference system. While the vision community generally makes use of checkerboard targets for camera calibration, the use of planar grids containing lines, dots or crosses is rather common in planar velocimetry calibration and for experimental fluid mechanics applications [13]. In either case, feature extraction algorithms recover the marker positions from the images and assign proper world (physical) coordinates to them. For the calibration tests described herein, grids of regularly spaced dots were used. Due to the symmetry of circular dots a detection scheme is easy to implement as well as more robust in off-axis or rotated viewing arrangements when compared to a grid of crosses or to a line grid, which require more elaborate detection schemes [14]. Experience has shown that, for the markers detection algorithms to work properly, the markers need to have a diameter of at least four/five pixels on the IR image.

The relative position between the calibration target and the observed object (model) has to be known. The target has to be moved along the whole volume in which the model will be situated, in order to acquire images in different known positions for the calibration and to map the observed volume. The positions of the control points are found within the images as the barycentre of the dots, evaluated using a threshold of the measured signal and, consequently, are known.

Once the space position and the image position of the control points are known, it is possible to apply the camera calibration algorithm. As proposed by Heikkila [12] the first trial value for perspective projection parameters is evaluated by a linear algorithm [15] which produces a first estimation. All the calibration constants are then found with a minimization of the root mean square difference between the known image coordinates of the control points and their image coordinates computed in accordance with the camera model. The minimization process is performed with the Levemberg-Marquardt method [16]. The convergence of the minimization process is very sensitive to the first trial value so the use of the first guess parameters obtained with the linear algorithm is very useful. This calibration approach is characterized by high speed, high accuracy and physically relevant camera parameters.

For a good convergence of the minimization process a number of markers higher than the minimum number necessary (14 control points) and a uniform distribution of the markers in the volume investigated are required. Typically 


\subsection{1/qirt.2016.160}

a good solution is obtained with a plane target with at least 14 control points and acquiring several images of the plane spanning the direction normal to the target in order to achieve the same spatial distribution of control points in the investigated volume.

It must be noticed that the design of a target should guarantee that the markers are well visible from the IR camera both in terms of dimensions and in terms of their features. For the applications in IR thermography described in this work, the contrast between the markers and the plane on which they are placed can be reached either using different emissivity or devising some method to keep the makers at a different temperature from the plane. Given the dependence of the target design on the particular experiment, detailed descriptions of the design procedures is postponed to the application section.

\subsection{D Surface temperature reconstruction}

The first step of temperature reconstruction is the generation of the mesh on the surface of the object. The availability of an accurate surface grid is an important requirement, which may be achieved either by in-house or by commercially available software. The mesh spacing has to be chosen to be just slightly higher than the spatial resolution of the IR camera, to avoid both loss of information and useless extra points (adding no further information but causing heavier computational load). The computed camera model constants are used to establish a correspondence between the points of the mesh and the pixels of the IR image. In that way it is possible to project the mesh points on the IR images and to evaluate the signal measured by the IR camera with an interpolation.

On the mesh points is possible to evaluate the viewing angle, as done by Giordano and Astarita [17]. If the first order Taylor series expansion of the calibration function is considered, a displacement $\underline{\mathrm{ds}}$ in the image plane $\Pi$ can be expressed as the product of the derivate of the calibration function by an infinitesimal space displacement $\underline{\mathrm{dS}}$ :

$$
\underline{d s}=\left[\begin{array}{c}
d u \\
d v
\end{array}\right]=\left[\begin{array}{lll}
u_{X} & u_{Y} & u_{Z} \\
v_{X} & v_{Y} & v_{Z}
\end{array}\right] \cdot \underline{d S}=\left[\begin{array}{ccc}
u_{X} & u_{Y} & u_{Z} \\
v_{X} & v_{Y} & v_{Z}
\end{array}\right] \cdot\left[\begin{array}{c}
d X \\
d Y \\
d Z
\end{array}\right]
$$

In order to determine the local viewing angle an infinitesimal displacement vector $\underline{\mathrm{dS}}$ along the viewing ray of the camera can be imagined. Obviously the correspondent displacement $\underline{\mathrm{ds}}$ in the image plane is zero.

The solution of this equation gives the viewing ray direction $\underline{\mathrm{W}}$ as represented in figure 1 :

$$
\left.\frac{d X}{d Z}\right|_{\underline{d s}=\underline{0}}=\left.\frac{v_{Z} u_{Y}-v_{Y} u_{Z}}{v_{Y} u_{X}-v_{X} u_{Y}} \quad \frac{d Y}{d Z}\right|_{\underline{d s}=\underline{0}}=\frac{v_{Z} u_{X}-v_{X} u_{Z}}{v_{X} u_{Y}-v_{Y} u_{X}}
$$

Infact by choosing $d Z=1$ it is possible to determine the normalised vector of the viewing ray direction.

$$
\underline{W}=\left[\left.\left.\frac{d X}{d Z}\right|_{\underline{d s}=\underline{0}} \frac{d Y}{d Z}\right|_{\underline{d s}=\underline{0}} 1\right]
$$

With a scalar product of the viewing ray direction by the known normal unit vector $\underline{\mathrm{n}}$ on the object surface, the viewing angle $\theta$ on the object surface is easily evaluated.

$$
\theta=\cos ^{-1}\left|\frac{\underline{W} \cdot \underline{n}}{|\underline{W}|}\right|
$$

As said before, the surface emissivity depends from viewing angle. Once the viewing angle is estimated, it is possible to correct the emissivity value with a law that relates emissivity to the viewing angle. A model reported by Siegel and Howell [18] and laniro and Cardone [19], relates emissivity to the refraction index and to the extinction coefficient (the imaginary part of the complex index of refraction).

The value of the temperature for every grid point is finally evaluated according to Eq. (10) 


$$
T=\frac{B}{\ln \left\{\frac{\varepsilon(\theta) \cdot R}{\left[U_{D}-(1-\varepsilon(\theta)) \frac{R}{e^{B / T_{a m b}}-F}\right]}+F\right\}}
$$

\section{Optical calibration and experimental apparatus}

In order to obtain the 3D temperature map of the ceramic shell mold, the employed procedure involves two stages: the calibration and the measurement stages. In both stages, a FLIR IR Thermocamera S65 equipped with a $72 \mathrm{~mm}$ lens is used with a resolution of $2.5 \mathrm{~mm} / \mathrm{px}$. During the calibration stage, images of the calibration target are recorded as shown in figure $2 a$, while in the measurement stage images of the investigated ceramic shell mold are acquired as depicted in figure $2 \mathrm{~b}$.

a)

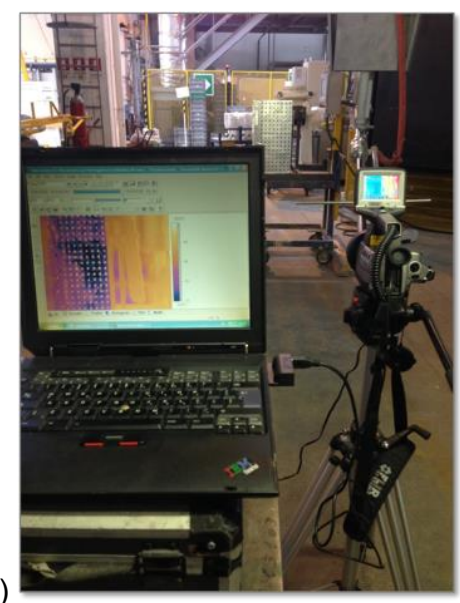

b)

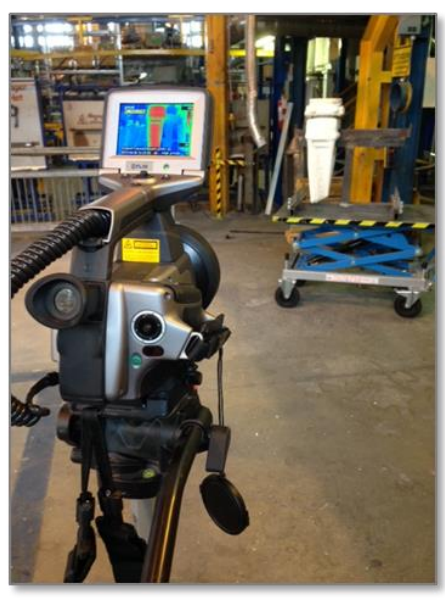

Fig. 2. Experimental apparatus during the a) calibration and b) experimental stage.

Focusing on the calibration stage, the main component is the calibration plate. This plate consists of: a wooden plate, a circuit printed board heater and a black-dotted aluminum foil. The wooden plate dimensions are $80 \times 80 \times 0.6 \mathrm{~cm}$ while the circuit printed board heater, glued on the wooden plate, is $60 \times 25 \mathrm{~cm}$. These two plates are wrapped by an aluminum foil (figure $3 a$ ) which is covered by a Cartesian grid of black dots in the region heated by a circuit printed board (figure $3 b)$. The black dots are necessary because their emissivity is much higher $(\epsilon \approx 0.9)$ than the aluminum one $(\epsilon \approx 0.3)$ allowing to visualize the control points in the IR images as required by the calibration procedure [11]. These black dots have a spacing of $35 \mathrm{~mm}$ and a diameter equal to $10 \mathrm{~mm}$ resulting in a resolution of $4 / 5$ pixels on the IR sensor, optimal for the calibration as suggested by Cardone et al. [11].

a)

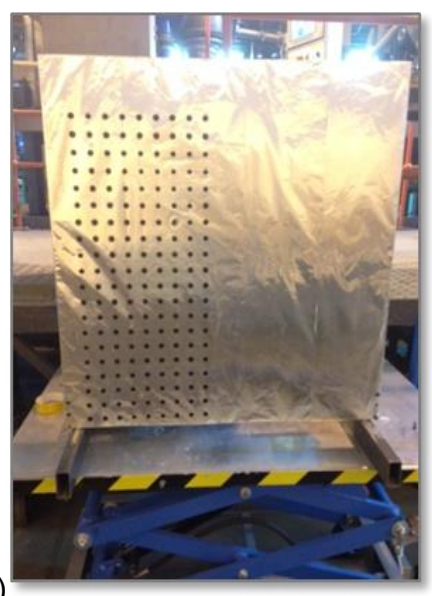

b)

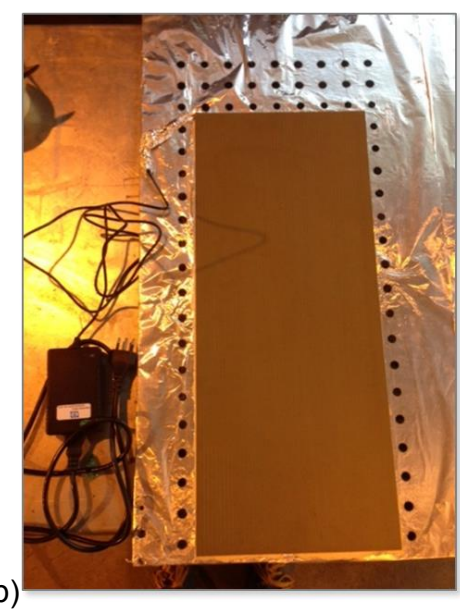

Fig. 3. Calibration plate. 


\subsection{1/qirt.2016.160}

Following the procedure illustrated in Section 2 (Cardone et al. [11]), the calibration plate sweeps the volume depth $(150 \mathrm{~mm})$ occupied by the investigated ceramic shell mold. The calibration plate is mounted on a rail and is moved by using an accurate sliding device; images of the target are acquired every $25 \mathrm{~mm}$. After determining the positions of the target dots within the images, it is possible to create a correspondence between the real-world coordinates of these markers and their position in the IR camera image plane. The camera model used in the present experiments is the same described in [11].

In order to reconstruct the 3D surface temperature maps, apart from the mapping function obtained with the optical calibration, the generation of a mesh on the surface of the investigated blade is necessary. The optical scanning is performed by using the ATOS ScanBOX 5120, as shown in figure 4a. The entire blade is reconstructed (figure 4b) with an accuracy of $0.01 \mathrm{~mm}$ and then discretized (figure $5 \mathrm{a}$ ) with a spacing between the sampled points of $5 \mathrm{~mm}$ which is greater than the IR camera resolution [11]. After the calibration and scanning process, the application of the camera model allows projecting the discretized points of the ceramic shell mold on the IR camera image, as shown in figure $5 \mathrm{~b}$. The temperature in the mesh points is evaluated from the IR images with an interpolation.

a)

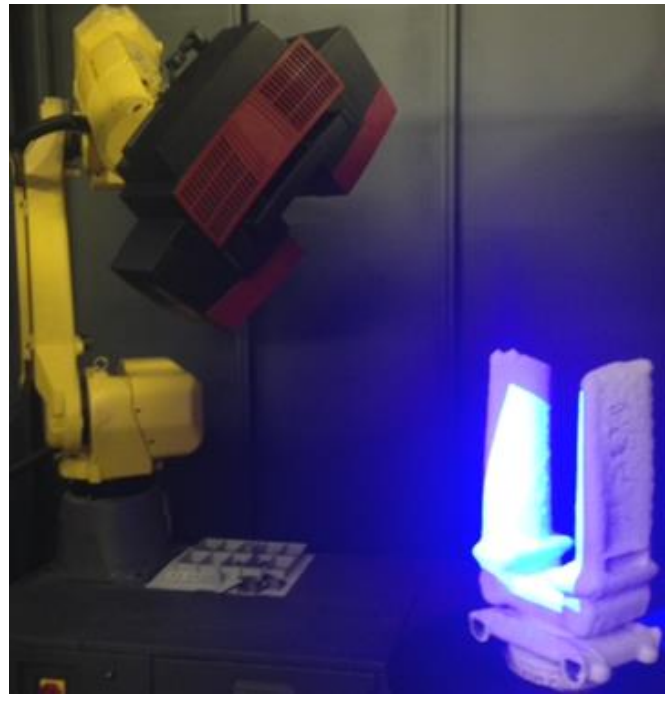

b)

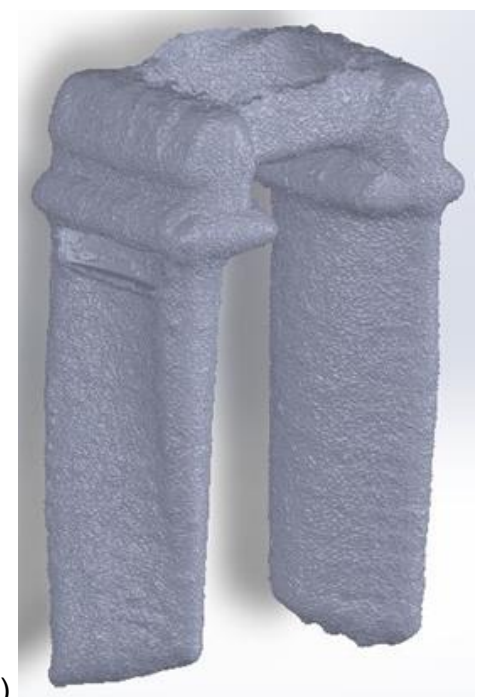

Fig. 4. a) Setup and b) output of the optical scanning of the investigated ceramic shell mold.

a)

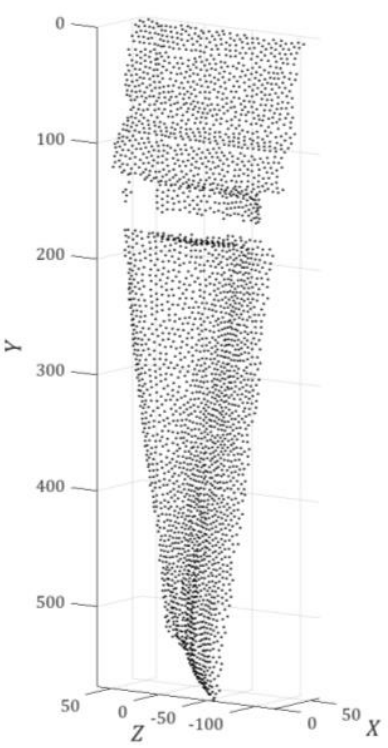

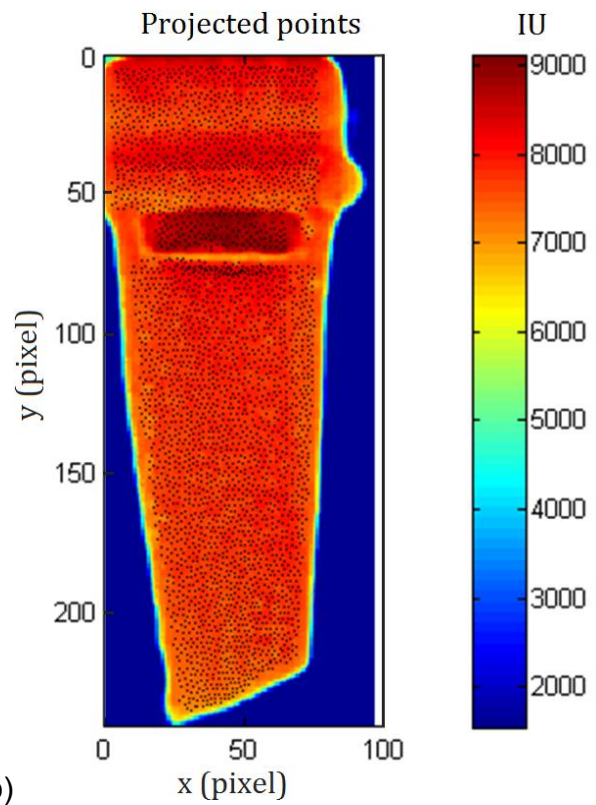

Fig. 5. ) 3D surface mesh of the investigated ceramic shell and b) its projection on the IR camera image plane (Axes values are in millimetres). 


\subsection{1/qirt.2016.160}

During the measurement stage, the ceramic shell mold is extracted from the preheating furnace and positioned in the same location where the calibration plate has been moved. The position of the ceramic shell mold with respect to the calibration plate is known. The IR acquisition of the heated ceramic shell mold begins after 30 seconds from the open of the pre-heating furnace. This time is chosen because it is approximately equal to the span of time necessary for the ceramic shell mold to pass from the pre-heating furnace to the high-temperature vacuum furnace.

\section{Results}

As previously explained, the $2 \mathrm{D}$ IR image captured by the IR camera allows for the reconstruction of the 3D surface temperature distribution by applying the camera model [11]. Such a procedure is performed individually for each of the three investigated parts of the ceramic shell mold: pressure side, leading edge and trailing edge.

In this way it is possible to see the real $3 D$ distribution of the mold temperature before it enters the hightemperature vacuum furnace for the casting. This temperature map is important because it is close to the initial boundary condition for the radiative heat transfer process which develops between the furnace and the shell during the casting.

In order to have a correct 3D temperature map reconstruction, the emissivity of each part of the ceramic shell mold has been evaluated. Indeed real objects almost never emit in an isotropic way and the emissivity coefficient $\epsilon$ is dependent on the angle $\theta$ (directional emissivity) between the direction of emission and the normal to the emitting surface (viewing angle). Since the ceramic shell mold is a highly three-dimensional geometry, the value of the directional emissivity has been taken into account for a correct evaluation of the surface temperature distribution for each of the three investigated parts: pressure side, leading edge and trailing edge. In figure 6 the angle $\theta$ (left) and the emissivity coefficient $\epsilon$ (right) for the pressure side are represented. It is possible to point out that the emissivity coefficient value starts decreasing in those regions where the angle $\theta$ becomes larger than approximately $50^{\circ}$. Once a correct evaluation of the emissivity coefficient has been performed over all the mold, the temperature distribution can be calculated.

In figure 7 the entire 3D temperature maps of the investigated ceramic shell mold, whose dimensions are $600 \mathrm{~mm}$ height, $250 \mathrm{~mm}$ large and $150 \mathrm{~mm}$ depth, are shown (black-coloured regions are non-measurements zones). In particular, the temperature distribution for each of the analysed parts (i.e. pressure side, the leading and trailing edge) is represented in figure 8 (left, centre and right respectively).

Regions of low temperature can be observed near the bottom of the pressure side part of the mold and along the leading and trailing edges. This is due to the natural convection and the radiative heat transfer. Indeed both the heat transfer kinds are definitely stronger in those regions because they are less affected by the thermal radiation from the adjacent parts and more exposed to the interaction with external colder ambient air. Indeed, these regions have a wider view angle on the external ambient, which allows a greater radiative heat transfer. Moreover, convective heat transfer is not reduced by the confinement of neighbouring regions.
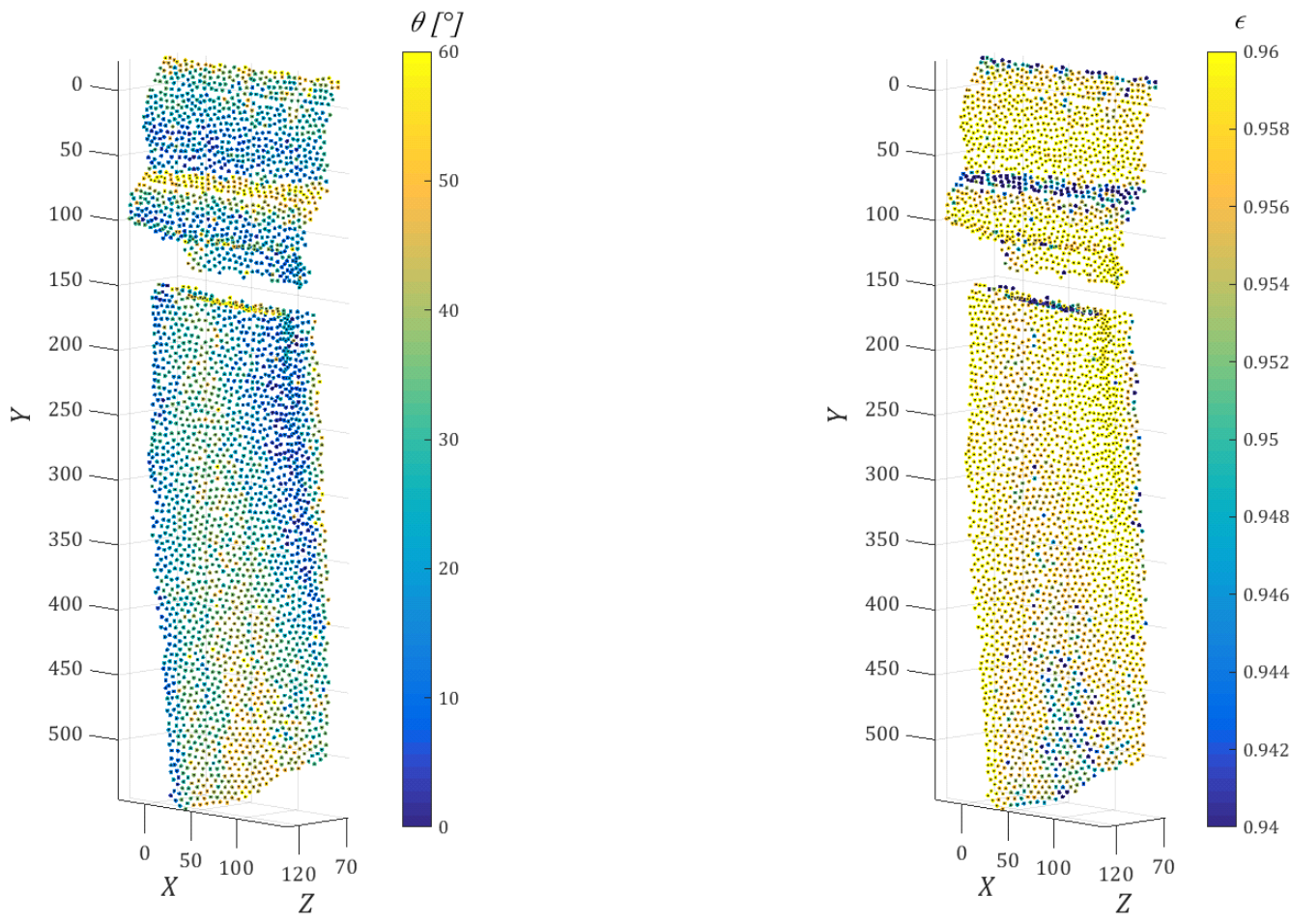

Fig. 6. The angle $\theta$ (left) between the direction of emission and the normal to the emitting surface and the emissivity coefficient $\epsilon$ (right). Axes values are in millimeters. 
Differently the high temperature regions are observed on the most part of the pressure side and, specifically, in the zones encircled in figure 7, hereinafter called as region I and II. Region I (the top one) has a higher temperature because it is confined by the presence of a protrusion on the bottom. from which a hot ascending flow is generated. This hot flow runs along the discussed region causing a decrease in the natural convection heat transfer. Moreover the protrusion is also present in the field of view of this region causing a reduction of the radiative heat transfer. Region II (the bottom one) attains such high values of temperature because it is a cavity inside the mold so it is confined by two protrusions (on the top and on the bottom). In this case the natural convection heat transfer drops off for the same reason previously explained while the radiative heat transfer is even more affected because the field of view of this region is more constrained by the presence of these two protrusions. Indeed the latter region shows temperature values which are much higher than those observed in the former one.

Looking at the temperature distribution along the shell height (corresponding to the blue axis in figure 7) on the pressure side part of the ceramic shell mold, it is possible to point out that it increases with the height considering the zone from the bottom of the shell until the region II. This is due to the natural convection transfer which decreases along the mold height, while the radiative heat contribution is approximately the same because no significant differences can be observed in the field of view.
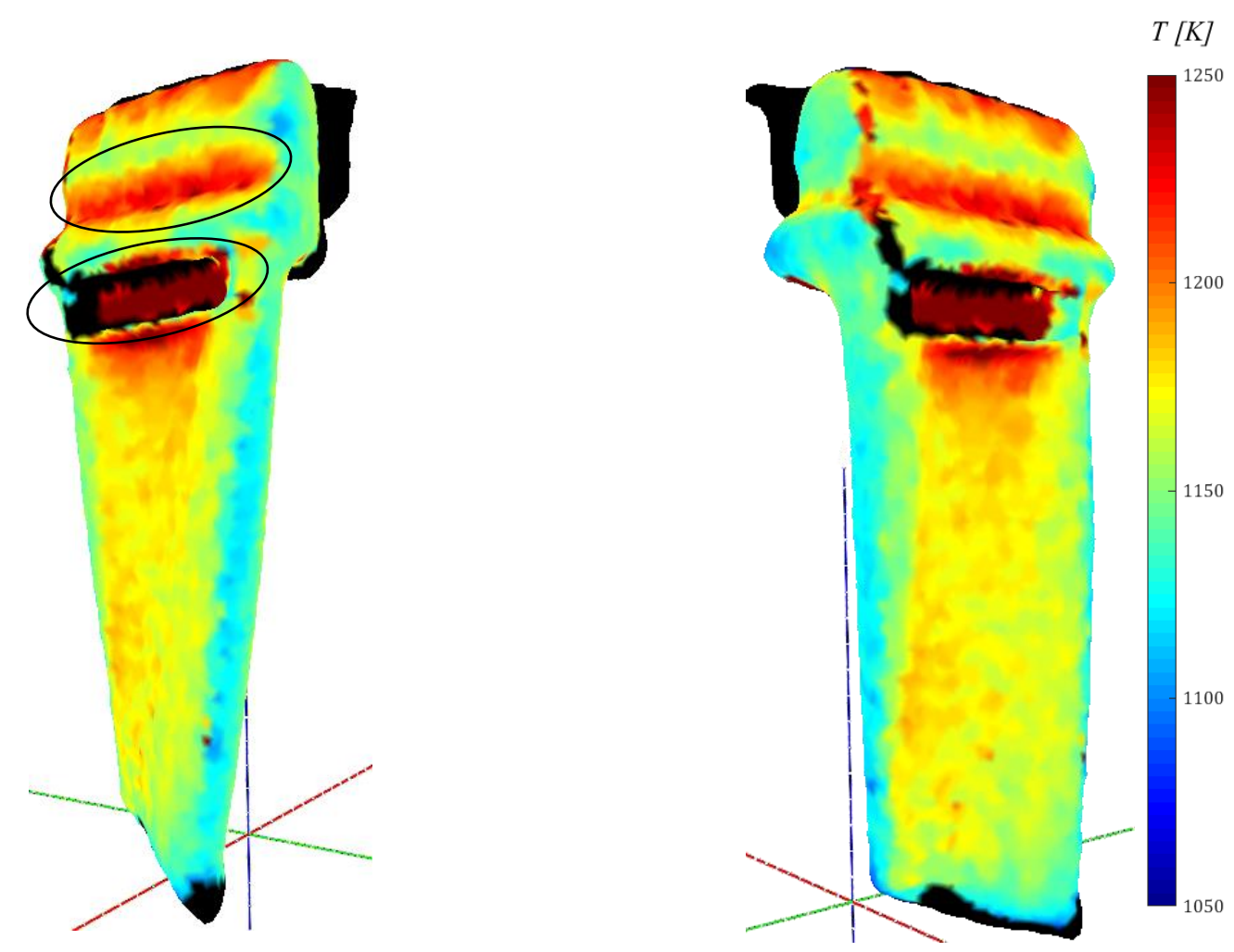

Fig. 7. 3D surface temperature map of the investigated ceramic shell mold (Black-coloured regions are nonmeasurements zones).

This difference of temperature distribution on the ceramic shell mold should be avoided because it deeply affects the cooling of the metal during the investment casting. Indeed, the difference between the starting temperature over the mold surface could causes mechanical damage of the blade, such as cricks, because of a different cooling behaviour. The leading and trailing edge are the most stressed part because they attain the lowest temperature values over the all investigated mold. These temperature values are rather different from the melted metal temperature leading to a high temperature gradient. Such a gradient can cause residual thermal stress in the blade which can be dangerous.

\section{Conclusions}

This paper reports on the surface temperature reconstruction of a ceramic shell mold in a specific stage of the investment casting process, namely the transportation between the preheating furnace and the high-temperature vacuum furnace. The temperature maps are obtained by means of the Infrared Thermography and then rebuilt onto the 3D surface by applying a camera calibration model. Both the optical calibration method and the experimental apparatus have been shown. The $3 \mathrm{D}$ temperature reconstruction technique has been applied separately for each part of the 
investigated mold: pressure side, trailing edge and leading edge. Then, an overall surface temperature distribution has been obtained by connecting the three individual ones. In order to improve the accuracy of 3D temperature reconstruction, the emissivity coefficient has been experimentally evaluated taking into account its variation with the emission angle. The reconstructed 3D surface temperature map shows that, after the exit from the preheating furnace, the initial temperature distribution of the shell mold changes drastically. Initially, the surface temperature is expected to coincide approximately with the preheating temperature, but, during the transportation, radiative and convective heat transfer occurs and leads to a non-uniform distribution. Regions of high temperature are observed in the pressure side and, in particular, in that zones where protrusions are present because they causes a decrease of both natural convection and radiative heat transfer. On the contrary, lower temperatures are detected in the trailing and leading edges and on the bottom of the pressure side because these regions are more exposed to the external ambient air and less influenced by the high-temperature zones of the mold itself.
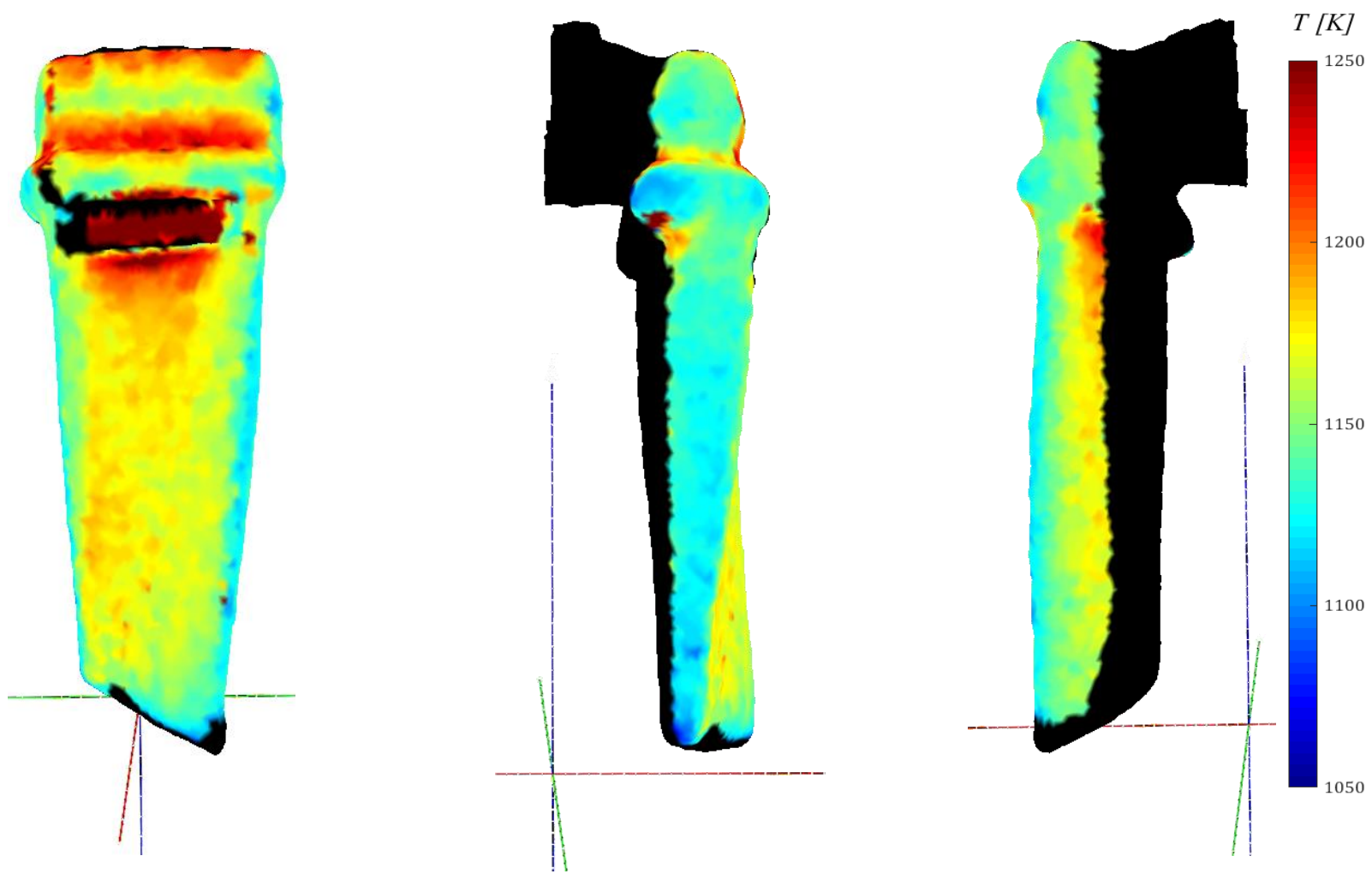

Fig. 8. 3D surface temperature map of pressure side (left), leading edge (centre) and trailing edge (right). (Black-coloured regions are non-measurements zones).

\section{Acknowledgment}

The authors gratefully acknowledge the financial support of MIUR which funds this work with the grant PON03PE_00111_1MATEMI.

\section{REFERENCES}

[1] Sabau A.S., "Numerical simulation of the investment casting process", Transactions of American Foundry Society, 2005.

[2] Shen Y.K., Liu J.J., Chang C.T., Chiu C.Y., "Comparison of the results for semisolid and plastic injection moulding process", International Communications in Heat and Mass Transfer, vol.29 (1), pp.97-105, 2002.

[3] Pattnaik S., Karunakar D.B., Jha P.K., "Developments in investment casting process - A review", Journal of Materials Processing Technology, vol.212, pp.2332-2348, 2012.

[4] Jones S., Yuan C., "Advances in shell moulding for investment casting", Journal of Materials Processing Technology, vol.135, pp.258-265, 2003.

[5] Browne D. J., O'Mahoney D., "Interface heat transfer in investment casting of aluminum alloys", Metallurgical and Materials Transactions A, vol.32 (12), pp.3055-3063, 2011. 


\subsection{1/qirt.2016.160}

[6] Konrad C.H., Brunner M., Kyrgyzbaev K., Völkl R., Glatzel U., "Determination of heat transfer coefficient and ceramic mold material parameters for alloy IN738LC investment castings", Journal of Materials Processing Technology, vol.211 (2), pp.181-186, 2011.

[7] Dong Y., Bu K., Dou Y., Zhang D., "Determination of interfacial heat-transfer coefficient during investmentcasting process of single-crystal blades", Journal of Materials Processing Technology, vol.211(12), pp.21232131, 2011.

[8] O'Mahoney D., Browne D. J., "Use of experiment and an inverse method to study interface heat transfer during solidification in the investment casting process", Experimental Thermal and Fluid Science, vol.22 (3), pp.111122, 2000.

[9] Carlomagno G. M., Cardone G., "Infrared thermography for convective heat transfer measurements", Experiments in fluids, vol.49 (6), pp.1187-1218, 2010.

[10] Astarita T., Carlomagno G. M., "Infrared Thermography for Thermo-fluid-dynamics", Springer Science \& Business Media, 2012

[11] Cardone G., laniro A., dello loio G., Passaro A., "Temperature maps measurements on 3D surfaces with infrared thermography”, Experiments in Fluids, vol.52, pp.375-385, 2012.

[12] Heikkilä J., Silven O., "A four-step camera calibration procedure with implicit image correction", Proceedings of the 1997 Conference on Computer Vision and Pattern Recognition (CVPR '97), pp.1106-1112, 1997.

[13] Willert C., "Assessment of camera models for use in planar velocimetry calibration", Experiments in Fluids, vol.41(1), pp.135-143, 2006.

[14] Ehrenfried K., "Processing calibration-grid images using the Hough transformation", Measurements Science and Technology, vol.13, pp.975-983, 2002.

[15] Abdel-Aziz Y.I., Karara H.M., "Direct linear transformation into object space coordinates in close-range photogrammetry", Proceedings of Symposium on Close-Range Photogrammetry, pp.1-18, 1971.

[16] Marquardt D., "An algorithm for least-squares estimation of nonlinear parameters", SIAM Journal of Applied Mathematics, vol.11(2), pp.431-441, 1963.

[17] Giordano R., Astarita T., "Spatial resolution of the Stereo PIV technique", Experiments in Fluids, vol.46(4), pp.643-658, 2009.

[18] Siegel R., Howell J.R., "Thermal Radiation Heat Transfer", 3rd ed. Hemisphere, Washington.

[19] laniro A., Cardone G., "Measurement of surface temperature and emissivity with stereo dual-wavelength IR thermography", Journal of Modern Optics, vol.57(18), pp.1708-1715, 2010. 